\title{
Elephant: uma aplicação móvel de estímulo cognitivo para pacientes de Alzheimer
}

\author{
Mirlandio S. S. Júnior ${ }^{1}$, Miguel V. Oliveira ${ }^{2}$, Márcia C. P. Martins ${ }^{1}$, Emmerson S. R. Silva ${ }^{1}$ \\ ${ }^{1}$ Departamento Acadêmico de Informação e Comunicação - Instituto Federal de Educação, \\ Ciência e Tecnologia do Amazonas (IFAM) - Manaus - AM - Brasil \\ ${ }^{2}$ Núcleo de Computação - Universidade do Estado do Amazonas (UEA) - \\ Manaus - AM - Brasil \\ \{pblatd, migvanderlei\}@gmail.com, marcia.martinseifam.edu.br, emmsr2004@gmail.com
}

\begin{abstract}
Alzheimer's disease is a dementia that affects about 50 million people around the world, and is characterized by gradual loss of memory and other intellectual abilities. This article presents a mobile application that stimulates the practice of exercises to develop cognitive aspects, such as logical reasoning and memory, combined with physical activities. The application still under development will have its effectiveness tested with a group of volunteer patients and their responsible doctors at a municipal elderly care institution.
\end{abstract}

Resumo. A Doença de Alzheimer é uma doença demencial que acomete cerca de 50 milhões de pessoas, e é caracterizada pela perda gradual de memória e outras capacidades intelectuais. Este artigo apresenta uma aplicação móvel que estimula a prática de exercícios para desenvolver aspectos cognitivos, como raciocínio lógico e memória, além de atividades físicas. A aplicação ainda em desenvolvimento terá sua eficácia testada com um grupo de pacientes voluntários e seus médicos responsáveis em uma instituição municipal de cuidados ao idoso.

\section{Introdução}

De acordo com dados do Instituto Brasileiro de Geografia e Estatística (IBGE), a proporção da população idosa (acima de 65 anos) tem crescido de forma constante nos últimos anos, representando hoje $9,52 \%$ da população total, com a estimativa de atingir a marca de $21,49 \%$ nos próximos 20 anos. Desta maneira, implica-se ter iniciado, proporcionais ao crescimento, transformações na incidência e prevalência das doenças crônicas e incapacitantes que atingem indivíduos de idade mais avançada [Barreto et al 2015].

Dentre as doenças crônicas e incapacitantes, destaca-se a Doença de Alzheimer (DA), uma doença demencial que compromete a integridade física, intelectual e social dos pacientes, geralmente, idosos. Caracterizada por múltiplos défices cognitivos, onde envolve o comprometimento da memória e perda progressiva da capacidade funcional [Cruz et al 2015]. Segundo a Alzheimer's Disease International, cerca de 50 milhões de pessoas no mundo estão vivendo com Alzheimer, sendo 58\% residentes de países em desenvolvimento. 
O presente artigo objetiva apresentar um aplicativo móvel chamado Elephant, que conta com exercícios categorizados em Cognitivos, Lógicos, Lembranças Pessoais e Atividades Físicas. O foco da aplicação é estimular o intelecto e a sociabilização do paciente, a fim de amenizar os impactos do progresso da doença para melhorar a qualidade de vida e impulsionar a ação dos métodos convencionais.

A primeira etapa do trabalho consistiu em estudo da bibliografia relacionada e de aplicações voltadas para pessoas acometidas com Alzheimer. Posteriormente, foram realizadas reuniões com profissionais de uma Fundação especializada em cuidados de pessoas idosas, para discutir a viabilidade da ferramenta e planejar testes com um grupo de voluntários para a validação da mesma. Logo, foi realizada a modelagem do software e o desenho da interface do sistema, para assim, dar início à codificação do aplicativo.

\section{Uso da tecnologia como estímulo cognitivo}

O estudo de caso de Cruz et al (2015) afirma que o uso de técnicas não farmacológicas, como a estimulação cognitiva com o idoso podem auxiliar na estabilização ou resultar até mesmo em leves melhoras dos défices cognitivos e funcionais. De acordo com Carvalho et al, (2016) em revisão sistemática da literatura, o estímulo cognitivo e de raciocínio lógico do paciente de DA auxiliam a farmacoterapia a amenizar os sintomas psicológicos e comportamentais da doença. Para Brito et al (2017), a exibição de fotografias, vídeos e músicas que remetem a experiências vivenciadas pelos pacientes são exemplos de ferramentas estimulantes para a cognição humana e a promoção da saúde mental. Além disso, a prática de atividades físicas e o convívio social são essenciais para a manutenção da qualidade de vida do indivíduo, conforme atestado por Kamada et al (2018). A partir dos três últimos trabalhos citados, foram elaboradas as quatro categorias de exercícios apresentados na aplicação.

Nos últimos anos, houve um avanço incorporado de novas tecnologias associadas à melhora da prevenção, diagnóstico e tratamento de doenças na área da saúde [Amorim et al 2010]. A quantidade de trabalhos científicos desenvolvidos no tema específico da utilização de jogos cognitivos para auxiliar no tratamento da DA e de outros usos da tecnologia como método não farmacológico é escassa. Portanto, o uso de uma aplicação móvel para estimular um paciente de DA a praticar exercícios cognitivos diariamente, além de convidá-lo a praticar atividades físicas que permitam a interação com outras pessoas pode ser caracterizado como uma inovação.

\section{O aplicativo Elephant}

Dentre os requisitos funcionais levantados para a aplicação, destacam-se: Realizar cadastro, Alterar dados, Registrar questão de memória, Responder questões, Pontuar questões e Consultar estatísticas. Já os requisitos não funcionais essenciais listados para o software são: Conexão com o servidor Firebase, Conexão com o banco de dados em nuvem, Funcionalidades de simples acesso. Destaca-se que a usabilidade da Interface de Usuário visa criar uma experiência acessível para pessoas na terceira idade.

Na Figura 1 estão representadas capturas de tela da aplicação, mostrando a tela de início, com as opções de autenticação e registro. Em seguida, a tela de autenticação, na qual o usuário informa e-mail e senha cadastrados para então ter acesso aos exercícios propostos em suas quatro categorias, além de poder visualizar dados do perfil e gráficos de progresso gerados pela aplicação. 

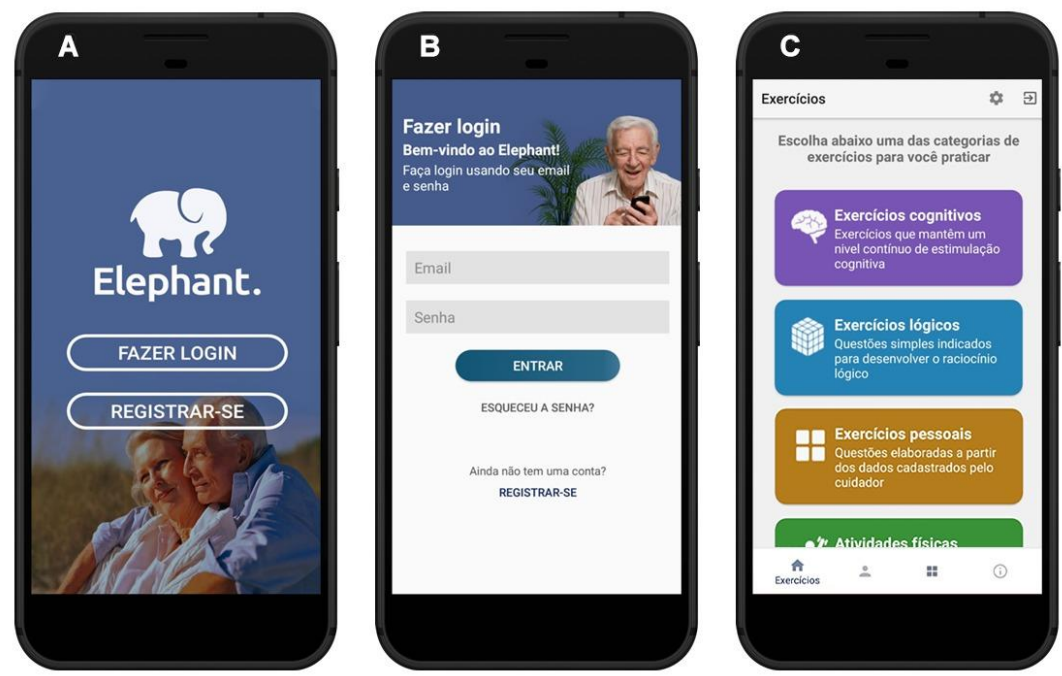

Figura 1. Capturas de tela do Elephant, mostrando tela inicial (A), autenticação por endereço de e-mail e senha (B) e a tela principal da aplicação em desenvolvimento $(\mathrm{C})$.

De acordo com a categoria selecionada, o usuário é direcionado para a tela de apresentação do exercício escolhido (Figura 2), onde é apresentada uma breve explicação acerca da categoria de exercício escolhida. Ao iniciar o exercício (Figura 2), são mostradas quatro alternativas de respostas, sendo apenas uma correta, nos moldes dos exercícios cognitivos mais populares. Objetivando estimular o usuário por meio do desafio, ao término da prática, o desempenho é contabilizado e a aplicação exibe uma mensagem com o número de questões respondidas corretamente.

A partir desses registros, o aplicativo gera um gráfico do desempenho geral na tela de Perfil (Figura 2), exibindo o percentual de exercícios completos, respondidos corretamente e questões não respondidas, considerando o número de exercícios propostos como a soma total, independente do status de completo ou não.
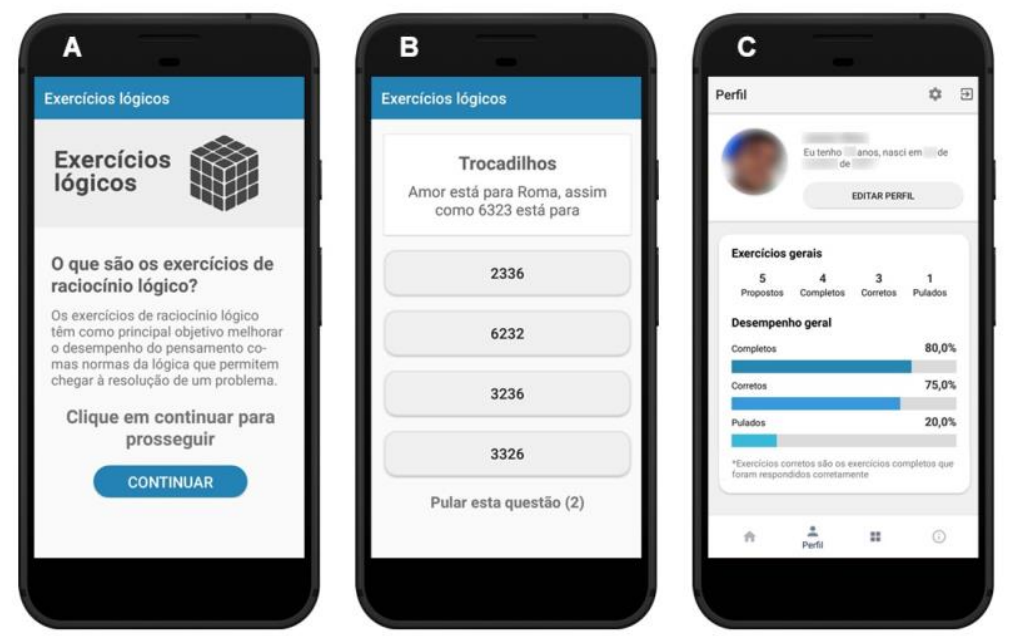

Figura 2. Capturas de tela exibindo a tela de Exercícios Lógicos e sua descrição (A), um exercício sendo realizado (B) e o perfil do usuário, com informações e estatísticas de progresso nos desafios (C). 


\section{Trabalhos futuros}

A validação da eficácia da ferramenta ocorrerá em duas etapas com um grupo de pacientes voluntários, cuidadores e médicos responsáveis da Fundação Doutor Thomas. A primeira consiste em uma avaliação da usabilidade da interface de usuário para o público idoso. Já a segunda tem o objetivo de monitorar o uso da aplicação como ferramenta de estímulo das capacidades cognitivas durante um período mínimo de três meses, com o apoio dos médicos da Fundação e o aval da comissão de ética.

A partir disso, será realizada coleta de resultados e da opinião dos pacientes acerca da aplicação, por meio de questionários, além da observação dos médicos responsáveis a respeito dos aspectos de lucidez e melhora nas capacidades cognitivas dos pacientes da DA. Dessa forma, a eficácia da aplicação móvel como ferramenta de estímulo mental como método não farmacológico para melhorar a qualidade de vida dos indivíduos afetados pela Doença de Alzheimer, abrindo precedentes para o uso de tecnologias móveis no tratamento de doenças demenciais.

\section{Referências}

IBGE, Projeção da população do Brasil e das Unidades da Federação. Instituto Brasileiro de Geografia e Estatística. Dados para consulta. Disponível em: $<$ https://www.ibge.gov.br/apps/populacao/projecao/>. Acesso em: 24 de fevereiro de 2019.

Barreto, M.S., Carreira, L. e Marcon, J. (2015) "Envelhecimento populacional e doenças crônicas: Reflexões sobre os desafios para o Sistema de Saúde Pública", em: Revista Kairós Gerontologia v. 18, n. 1, p. 325-339, São Paulo.

Cruz, T.J.P., Sá, S.P.C., Lindolpho, M.C e Caldas, C. P. (2015) "Estimulação cognitiva para idoso com Doença de Alzheimer realizada pelo cuidador", em: Revista Brasileira de Enfermagem v. 68, n. 3, p. 510-516, Brasília.

Carvalho, P. D. P., Magalhães, C. M. C., e Pedroso, J. D. S. (2016). "Tratamentos não farmacológicos que melhoram a qualidade de vida de idosos com doença de Alzheimer: uma revisão sistemática", em: Jornal Brasileiro de Psiquiatria, 65(4), 334-339, Rio de Janeiro.

Brito, L. P., Guimarães, M. S., Santana, L. S., e Araújo, P. O. (2017). “Exercícios de memória: uma estratégia para a promoção da saúde dos idosos", em: Anais V CIEH, V. 1, Campina Grande.

Kamada, M., Clemente, J. S., Monteiro, A. D. F. F., de Barros, L. V. G., Helene, A. H. E., e Morato, D. M. (2018). "Correlação entre exercício físico e qualidade de vida em pacientes com doença de Alzheimer", em: Revista da Sociedade Brasileira de Clínica Médica, 16(2), 119-122. São Paulo.

Alzheimer's Disease International. Federação Internacional de Associações pela Doença de Alzheimer. Dados e estimativas acerca da doença. Disponível em: $<$ https://www.alz.co.uk/research/statistics>. Acesso em: 05 de março de 2019.

Amorim, F. F., Ferreira Júnior, P. N., Faria, E. R., e Almeida, K. J. Q. D.. (2010) "Avaliação de tecnologias em saúde: contexto histórico e perspectivas", em: Comunicação em ciências da saúde, v. 2, n. 4, p. 343-348, Brasília. 